\title{
Incorporation of Proteins with Different Isoelectric Points into Biomimetic Ca-P Coatings: a New Approach to Produce Hybrid Coatings with Tailored Properties

\author{
H. S. Azevedo ${ }^{1,2, a}$, I. B. Leonor ${ }^{1,2}$, R. L. Reis ${ }^{1,2}$ \\ Campus de Gualtar, 4710-057 Braga, Portugal \\ ${ }^{2}$ Department of Polymer Engineering, University of Minho, Campus de Azurém, 4800-058 \\ Guimarães, Portugal \\ ahazevedo@dep.uminho.pt
} \\ 13B's Research Group - Biomaterials, Biodegradables and Biomimetics, University of Minho,
}

Keywords: biomimetic coatings, calcium phosphate, proteins, isolectric point, hybrid

\begin{abstract}
This work aims to study the effect of incorporating proteins with different isoelectric points (pI) on the structure, composition and morphology of biomimetic calcium-phosphate (Ca-P) coatings. For that, bovine serum albumin (BSA) and lysozyme, having respectively acidic and basic pIs, were used as model proteins. It was observed that the incorporation of positively charged proteins, such as lysozyme, was able to significantly change the structure of the coatings, possibly due to the preferential interactions between the protein and negatively charged phosphate ions. These results indicated that proteins with different characteristics can be incorporated into biomimetic Ca-P coatings in order to obtain a hybrid coating and at the same tailoring their properties.
\end{abstract}

\section{Introduction}

In Nature, there are many examples of structures where minerals are complexed with organic molecules to form hybrid materials, including for example the structure of vertebrates laminar bone [1]. Biomineralization is a highly complex event involving the selective recognition and deposition of calcium ions mediated by proteins, followed by the formation of a mineral phase comprising crystallites with specific orientation and morphology [2]. The mechanism through which organic macromolecules control the synthesis, construction and organization of inorganic mineral is, however, still not clear. Proteins, through their unique and specific interactions with other macromolecules and inorganic compounds, control biological structures and functions in organisms. In this sense, specific proteins may be used, as templating or enzymatic agents, to control materials assembly and modulate the structure of inorganic surfaces such as calcium-phosphate (Ca-P) coatings. For instances, bioactive proteins can be directly integrated in the structure of biomimetic $\mathrm{Ca}-\mathrm{P}$ coatings, maintaining their conformation close to their native form, as they are generated under physiological conditions, and thus improve the functionality of the inorganic layer at the implant interface.

In this study, bovine serum albumin and lysozyme, having respectively acidic and basic isoelectric points $(\mathrm{pI})$, were incorporated into biomimetic Ca-P coatings with the aim of investigating the effect of protein $\mathrm{pI}$ on the structure, composition and morphology of the coatings.

\section{Materials\&Methods}

The procedure used to produce Ca-P coatings on the surface of the polymeric substrate (a 50/50 wt $\%$ blend of corn starch with poly(ethylene-vinyl alcohol) copolymer - SEVA-C) was based on a biomimetic methodology developed by Kokubo et al. [3] and further adapted by Reis et al. [4]. Briefly, a bioactive glass 45S5 $\left(45 \mathrm{SiO}_{2}, 24.5 \mathrm{CaO}, 24.5 \mathrm{Na}_{2} \mathrm{O}\right.$ and $6.0 \mathrm{P}_{2} \mathrm{O}_{5}$ in wt. \% - Novamin ${ }^{\circledR}$, NovaMin Technology Inc., Florida, USA) was used as nucleating agent. Control specimens were soaked in a simulated body fluid (SBF) solution, which has ion concentrations $\left(\mathrm{Na}^{+} 142.0, \mathrm{~K}^{+}\right.$5.0, 
$\mathrm{Ca}^{2+} 2.5, \mathrm{Mg}^{2+} 1.5, \mathrm{Cl}^{-} 147.8, \mathrm{HCO}_{3}{ }^{-} 4.2, \mathrm{HPO}_{4}{ }^{2-} 1.0, \mathrm{SO}_{4}{ }^{2-} 0.5 \mathrm{mM}$ ) [5] nearly equal to those of the human blood plasma, at $36.5^{\circ} \mathrm{C}$ and $\mathrm{pH}=7.40$. Another set of experiments was carried out by soaking the polymeric substrate in SBF solution containing bovine serum albumin $(\mathrm{BSA}, \mathrm{pI}=4.7)$ or lysozyme ( $\mathrm{pI}=11.0$ ), at a concentration of $1 \mathrm{mg} / \mathrm{mL}$, during the growth stage. The obtained Ca-P coatings were characterised by scanning electron microscopy coupled with energy dispersive spectroscopy (SEM/EDS), Fourier transform infrared spectroscopy (FTIR) and thin-film X-ray diffraction (TF-XRD). The incubation solutions were analysed by induced coupled plasma (ICP) spectroscopy in order to follow the evolution of $\mathrm{Ca}, \mathrm{P}, \mathrm{Si}$ and $\mathrm{Na}$ concentrations at different stages of the formation of the Ca-P film.

\section{Results}

The TF-XRD patterns of the polymeric surface for the various studied conditions are shown in Figure 1. Relatively weak reflections peaks, which are ascribed to a partially amorphous apatite-like phase (ASTM JCPDS 9-432) could be detected on the specimen surface after 7 days of immersion in the growth stage. Furthermore, the typical peaks of the polymeric substrate completely disappeared after the coating, as compared to uncoated substrate, which reinforces the formation of a Ca-P layer on the polymeric surface. The presence of proteins does not change significantly the coating crystallinity. However, when lysozyme was incorporated, it was also detected the presence of the octacalcium-phosphate (OCP) phase (ASTM JCPDS 26-1056).

FTIR analysis of the coatings (Figure 1) showed the presence of bands associated with apatite phosphate groups, which are in agreement with the TF-XRD results. The band corresponding to amide I, characteristic group of protein molecules, could be also detected in the IR spectra when the proteins were added to the $1.5 \mathrm{SBF}$, which indicated the efficient incorporation of the proteins into the coatings.
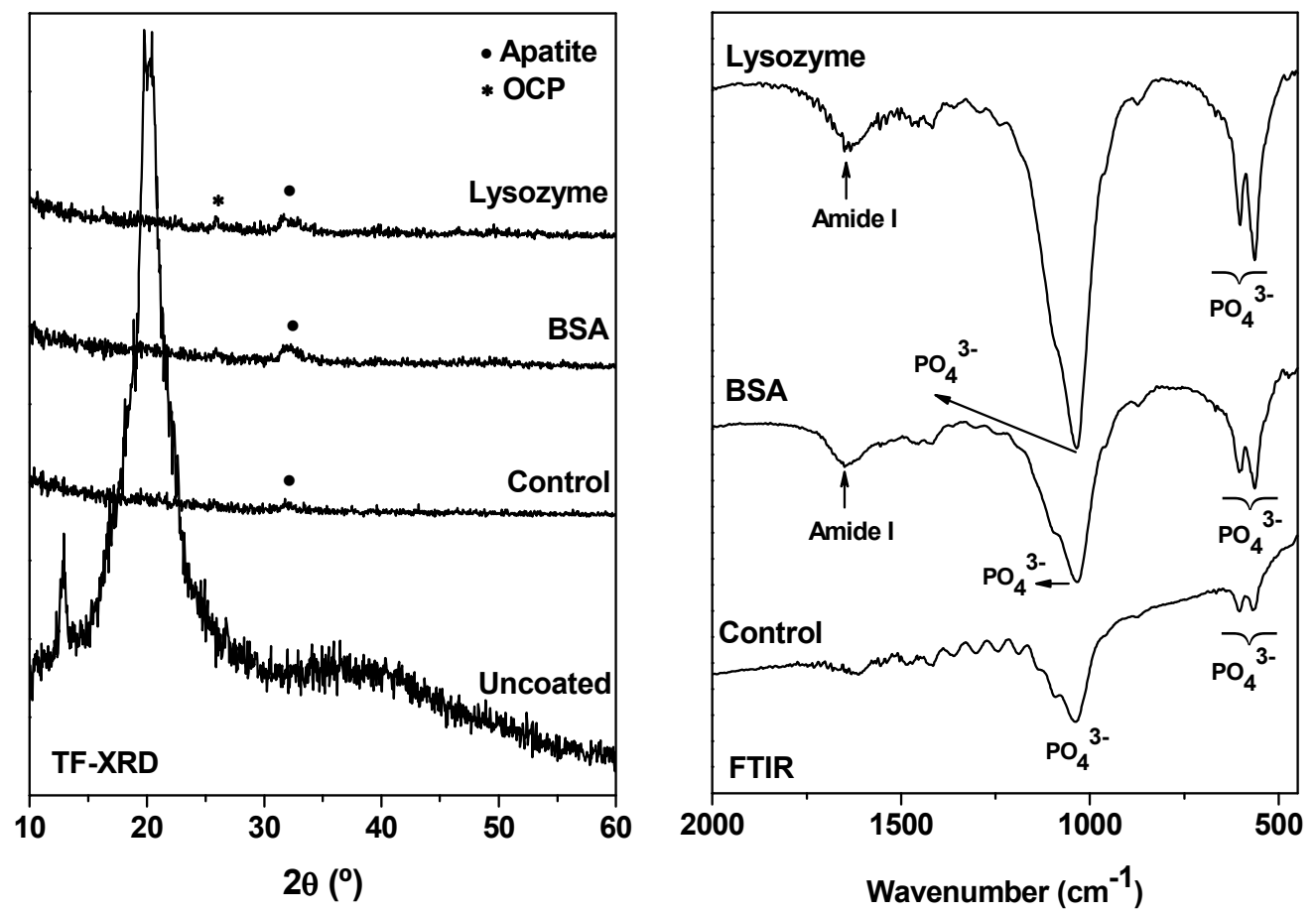

Figure 1: TF-XRD patterns and FTIR spectra of the Ca-P coatings produced on the surface of the polymeric substrate after 7 days of immersion in $1.5 \mathrm{SBF}$ during the growth stage for the various studied conditions

Figure 2 shows the SEM micrographs of the Ca-P coatings obtained for the various studied conditions and for different periods of immersion in 1.5 SBF. The incorporation of BSA $(\mathrm{pI}=4.7)$ and lysozyme $(\mathrm{pI}=11)$ leads to the formation of a Ca-P layer with different morphologies. The incorporation of lysozyme at the growth stage leads to the formation of plate-like crystals (Figure 2-c), instead of 
needle-like as it was observed for the control and for BSA (Figure 2-a, b). The nature of the interaction between the inorganic phase and the proteins is complex and poorly understood. Proteins can induce modifications on the crystal morphology by changing the relative growth rates of different crystal faces through molecular-specific interactions with particular surfaces.
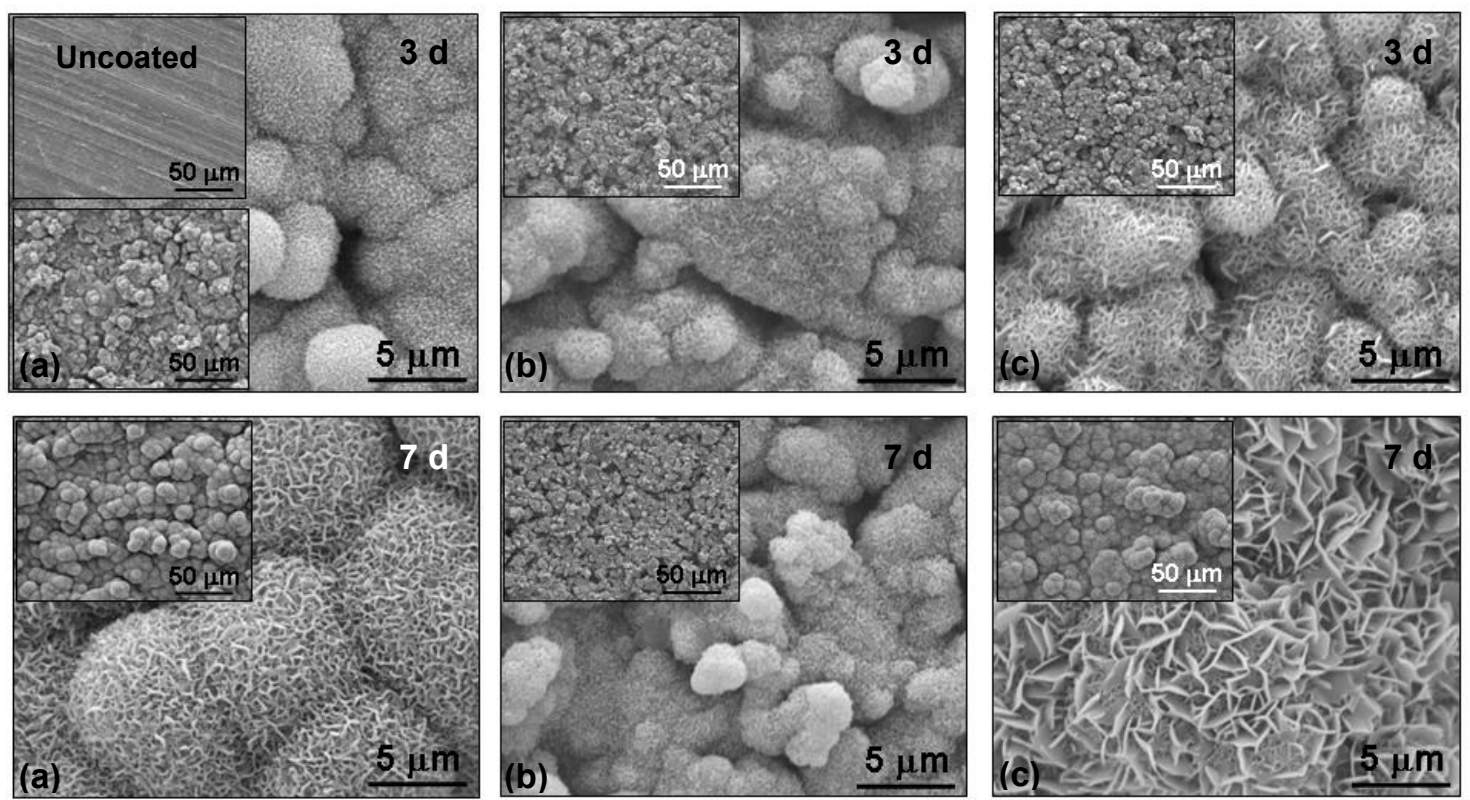

Figure 2: SEM micrographs of the polymeric surface after 3 and 7 days of immersion in 1.5 SBF during the growth stage showing the morphology of the Ca-P coatings obtained in the different studied conditions: (a) control, (b) BSA and (c) lysozyme incorporated at concentration of $1 \mathrm{mg} / \mathrm{mL}$ in the growth stage

At physiological $\mathrm{pH}, \mathrm{BSA}$ is negatively charged and may interact directly with calcium ions from the 1.5 SBF. In the case of lysozyme, which is carrying a positive charge at $\mathrm{pH} 7.4$, some preferential interactions between lysozyme and the negatively charged phosphate ions might be occurring. This phosphate affinity may have created locally phosphate-rich Ca-P nuclei, leading to the formation of phosphate rich, or calcium-deficient Ca-P crystals. By analysing the results presented in Figure 3, it can be concluded that there is a fast decrease of $\mathrm{Ca}$ and $\mathrm{P}$ concentrations in the first 3 days of immersion in 1.5 SBF and then gradually decrease as the soaking time increase. This decrease of $\mathrm{Ca}$ and $\mathrm{P}$ in solution is due to the formation of the $\mathrm{Ca}-\mathrm{P}$ layer which is due to the consuming of calcium and phosphate from the SBF solution.
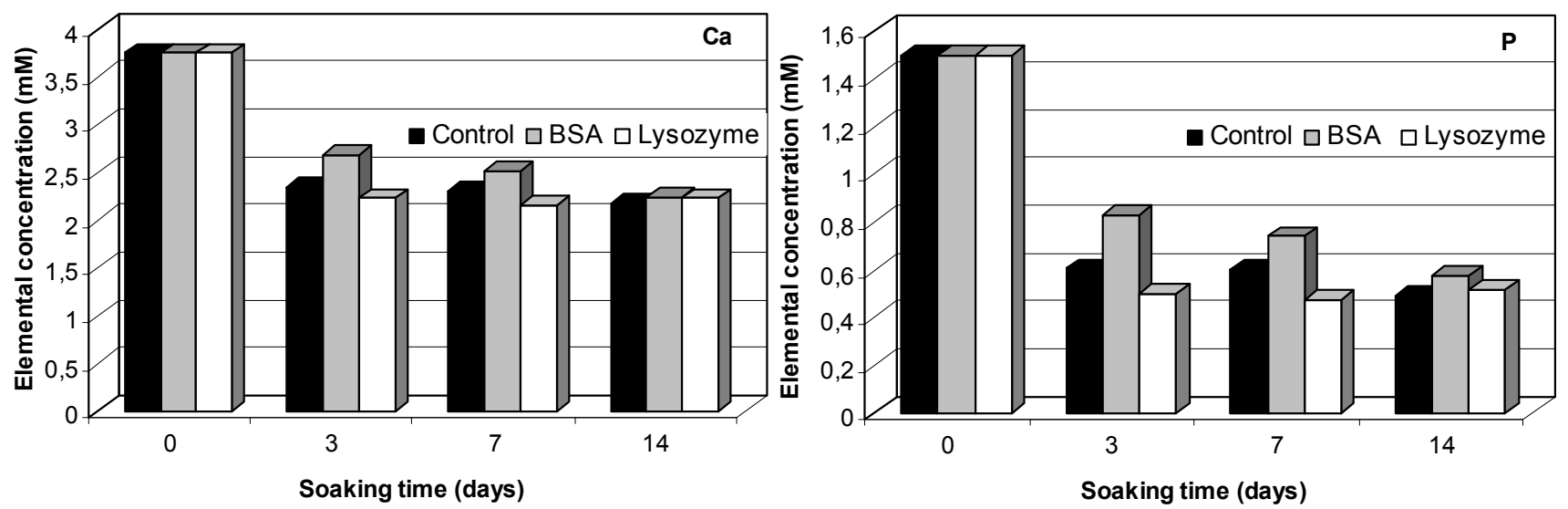

Figure 3: Evolution of $\mathrm{Ca}$ and $\mathrm{P}$ concentrations in 1.5 SBF as function of immersion time in the growth stage for the various studied conditions

However, when lysozyme was incorporated at the growth stage, the decrease in P concentration is more significant when compared with the control and BSA. This observation may confirm the 
preferential interactions between lysozyme and the negatively charged phosphate ions. Poumier et al. [6] showed that adsorption of lysozyme on synthetic hydroaxyapatite occurs through adsorption on negative ions like phosphate.

\section{Discussion and Conclusions}

Bone tissue can be viewed as a two-component composite material composed primarily of collagen and mineral, hydroxyapatite, in the form of needle like crystallites precipitated along the collagen fibrils. Therefore, when designing bone substitutes with outstanding properties, the implant should be not a single material but a combination of two materials. An example of such combination is the biomimetic coating technique, which has been developed for the formation of a Ca-P osteoconductive coating on implant surfaces with high mechanical properties. The good performance of Ca-P-coated implant depends on several factors such as the degradability of the coating and the variation in bond strength at the coating-implant surface. In contrast to plasma-spraying, biomimetic coating methodology, i.e., deposition of Ca-P coatings from SBF solution under physiological conditions, allow to produce coatings with tailored $\mathrm{Ca} / \mathrm{P}$ ratios (tailored resorbability), and good adhesion coating/substrate that will be able to induce stimulus on bone cells. This may be also achieved by delivering osteoinductive proteins able to induce local bone growth around the implant, using for instances, Ca-P coatings as a carrier.

In this study it was possible to observe that the incorporation of BSA and lysozyme changed the morphology of the Ca-P crystals. In fact, the incorporation of proteins in crystals normally leads to changes in the number, size and distribution of crystal aggregates. However, the extent of these effects depends on some parameters like the protein concentration and the way these biomacromolecules are present in solution (free or immobilized). One of the explanations for these results may be related with differences in the amino acid composition of the proteins, and in particular the number and orientation of certain amino acid residues exposed at the protein surface (protein conformation). These functional groups may be involved in the initial stages of mineral formation through complexation of calcium cations, which in turn attract phosphate anions. Depending on protein $\mathrm{pI}$, different electrostatic interactions will occur between proteins and ionic species in the solution, leading to the formation of a coating with different $\mathrm{Ca} / \mathrm{P}$ ratios. In fact, the incorporation of BSA and lysozyme (being negatively and positively charged at $\mathrm{pH} 7.4$, respectively) might have affected the initial step of the growth of a Ca-P film, by triggering calcium or phosphate ions from the fluid.

In this work, it was shown that proteins with different characteristics can be incorporated into biomimetic Ca-P coatings to obtain a hybrid coating and at the same time tailoring their properties. On the other hand, these hybrid coatings may present an additional benefit as they can simultaneously be used as a delivery system. The coating dissolution would allow the progressive release of the entrapped proteins directly to the tissue-implant interface. This approach could be used in controlled release applications of biologically relevant proteins (e.g. growth factors) and thus control cellular interactions that are important in hard tissue regeneration.

\section{References}

1. S. Weiner, L. Addadi and H.D. Wagner. Mater. Sci. Eng. C - Biomimetic and Supramolecular Systems 11 (2000): 1.

2. R. Lakshminarayanan, R.M. Kini and S. Valiyaveettil. Proc Natl Acad Sci U S A 99 (2002): 5155.

3. Y. Abe, T. Kokubo and T. Yamamuro. J: Mater. Sci: Mater. Med. 1 (1990): 233.

4. R.L. Reis, A.M. Cunha, M.H. Fernandes and R.N. Correia. J. Mater. Sci: Mater. Med. 8 (1997): 897.

5. T. Kokubo, H. Kushitani, S. Sakka, T. Kitsugi, T. Yamamuro. J. Biomed. Mater. Res. 24 (1990): 721.

6. F. Poumier, Ph. Schaad, Y. Haïkel, J.C. Vogel, Ph. Gramain. Colloids and Surfaces 7 (1996): 1.

Acknowledgements: Portuguese Foundation for Science and Technology through funds from the POCTI and/or FEDER Programmes (Grant SFRH/BPD/5744/2001), the European Union funded STREP Project HIPPOCRATES (NMP3-CT-2003-505758) and the European NoE EXPERTISSUES (NMP3-CT-2004-500283). 